\title{
A note on the correlation of classes.
}

\author{
By J. M. WhitTaker.
}

(Received 13th January 1927. Read 14th January 1927:)

1. Let $R$ be a $(1-1)$ relation between the members of two similar classes $A, B_{1}$. It correlates the members of a subclass $X$ of $A$ to the members of a certain subclass $Y$ of $B_{1}$ and thus defines a relation $\rho$ connecting $X$ and $Y$. It is clear that $\rho$ is a $(1-1)$ relation and that it has the property $(M)$. If $X_{1} \rho Y_{1}, X_{2} \rho Y_{2}$, then $X_{1} \subset X_{2}$ implies $Y_{1} \subset Y_{2}$.

It will be shown that

If $A_{\rho} B_{1} \subset B, B \sigma A_{1} \subset A$, there are subclasses $A_{0}, B_{0}$ of $A, B$ such that $A_{0} \rho B_{0}, B-B_{0} \sigma A-A_{0}$.

The proof consists in making a kind of Dedekind section of the subclasses $X$, and may be explained as follows.

If $X^{\prime}$ is defined by $X_{\rho} Y, B-Y_{\sigma} X^{\prime}$ we say that $X$ is a $U$ if $X, X^{\prime}$ overlap, and that $X$ is an $L$ if they do not. The subclass $A_{0}$ whose existence we wish to demonstrate is to be such that $A_{0}{ }^{\prime}=A-A_{0}$. i.e. it is to be an $L$ but as nearly as possible a $U$. Thus we might expect that there will be a largest $L$ and that this will be $A_{0}$. It is not difficult to prove that this is the case.

2. An immediate consequence of $(M)$ is the following lemma.

If $X_{1} \subset X_{2}$, then $X_{2}^{\prime} \subset X_{1}^{\prime}$.

Let $A_{0}=$ sum of all $L$ 's. ${ }^{1}$ Then in the first place

$$
A_{0} \subset A-A_{0}^{\prime} \text {. }
$$

For by the lemma $A_{\mathbf{0}}{ }^{\prime} \subset L^{\prime}$ for every $L$ and so

$$
L \subset A-L^{\prime} \subset A-A_{0}^{\prime} \text {. }
$$

1 There may be no L's, but this does not matter since the null class is counted as a subclass of $A$. It will be noticed that the proof depends only on the fact that $\rho, \sigma$ are $(1-1)$ relations with the property $(M)$, so that the theorem is true for any relations with these properties. Thus it is not necessary that the members of $X$ should be in $(1-1)$ relation with those of $Y$, nor that those subclasses of $B$ to which the subclasses of $A$ art correlated by $\rho$ should be all the subclasses of a certain part $B_{1}$ of $B$. 
Thus $A-A_{0}^{\prime}$ contains every $L$ and so it contains $A_{0}$. By (I) and the lemma

$$
\left(A-A_{0}{ }^{\prime}\right)\left(A-A_{0}{ }^{\prime}\right)^{\prime} \subset\left(A-A_{0}{ }^{\prime}\right) A_{0}{ }^{\prime}=0
$$

i.e. $A-A_{0}{ }^{\prime}$ is an $L$ and so is contained in $A_{0}$. But by (1) $A_{0}$ is contained in $A-A_{0}^{\prime}$. Thus

or

$$
\begin{aligned}
& A_{0}=A-A_{0}{ }^{\prime} \\
& A_{0}{ }^{\prime}=A-A_{0}
\end{aligned}
$$

which is the result stated.

3. An immediate corollary is the Schröder-Bernstein theorem.

If $A$ is similar to a part of $B$ and $B$ is similar to a part of $A$, then $A$ is similar to $B .^{1}$

Again let $A, B$ be simply ordered classes. We deduce that

If $A$ is ordinally similar to a part of $B$ and $B$ is ordinally similar to a part of $A$, then there is a part $A_{0}$ of $A$ which is ordinally similar lo a part $B_{0}$ of $B$ and such that $A-A_{0}$ is ordinally similar to $B-B_{0}$.

That the premisses of this proposition do not necessarily imply that $A$ is ordinally similar to $B$ is illustrated by the following trivial example. $A$ consists of the real numbers in $(0 \leqslant x \leqslant 1)$ together with the rational numbers in $(1 \leqslant x \leqslant 2), B$ of the real numbers in $(0 \leqslant y \leqslant 2)$. Then $A$ is not ordinally similar to $B$, but $A$ is ordinally similar to a part of $B$ by the relation $y=x$, and $B$ is ordinally similar to a part of $A$ by the relation $y=2 x . A_{0}, \mathrm{~B}_{0}$ are in this case the sets of rational numbers in $(0 \leqslant x \leqslant 2),(0 \leqslant y \leqslant 2)$. These sets are ordinally similar by the first relation, while the set of irrational numbers in $A$ is ordinally similar to the set of irrational numbers in $B$ by the second relation.

1.e. if $a, b$ are cardinal numbers, $a \leftarrow b$ and $b \leq a$ together imply $a=b$. 